\title{
Improvement of Salt Tolerance Using Wild Rice Genes
}

\author{
Ruidang Quan ${ }^{1,2 *}$, Juan Wang ${ }^{1,2}$, Jian Hui ${ }^{3}$, Haibo Bai ${ }^{3}$, Xuelian Lyu ${ }^{3}$, Yongxing Zhu ${ }^{3}$, \\ Haiwen Zhang ${ }^{1,2}$, Zhijin Zhang ${ }^{1,2}$, Shuhua $L_{i}{ }^{3 *}$ and Rongfeng Huang ${ }^{1,2 *}$ \\ ${ }^{1}$ Biotechnology Research Institute, Chinese Academy of Agricultural Sciences, Beijing, China, ${ }^{2}$ National Key Facility of Crop \\ Gene Resources and Genetic Improvement, Beijing, China, ${ }^{3}$ Ningxia Academy of Agriculture and Forestry Sciences, \\ Yinchuan, China
}

\section{OPEN ACCESS}

Edited by:

Jose M. Pardo,

Instituto de Bioquímica Vegetal y

Fotosintesis (CSIC), Spain

Reviewed by:

Keisuke Nagai,

Nagoya University, Japan

Ahmad Arzani,

Isfahan University of Technology, Iran

*Correspondence:

Ruidang Quan

quanruidang@caas.cn

Shuhua $L$

shuhua.1@163.com

Rongfeng Huang

rfhuang@caas.cn

Specialty section:

This article was submitted to

Plant Abiotic Stress,

a section of the journal

Frontiers in Plant Science

Received: 19 October 2017 Accepted: 27 December 2017

Published: 17 January 2018

Citation:

Quan R, Wang J, Hui J, Bai H, Lyu X,

Zhu Y, Zhang H, Zhang Z, Li S and

Huang $R$ (2018) Improvement of Salt

Tolerance Using Wild Rice Genes.

Front. Plant Sci. 8:2269.

doi: 10.3389/fpls.2017.02269
Salt stress causes significant reductions in rice production worldwide; thus, improving salt tolerance is a promising approach to meet the increasing food demand. Wild rice germplasm is considered a valuable genetic resource for improving rice cultivars. However, information regarding the improvement of salt tolerance in cultivated rice using wild rice genes is limited. In this study, we identified a salt-tolerant line Dongxiang/Ningjing 15 (DJ15) under salt-stress field conditions from the population of a salt tolerant Dongxiang wild rice $\times$ a cultivated rice variety Ningjing16 (NJ16). Genomic resequencing analysis of NJ16, DJ15 and Dongxiang wild rice revealed that the introgressed genomic fragments were unevenly distributed over the 12 chromosomes (Chr.) and mainly identified on Chr. 6, 7, 10, and 11. Using quantitative trait locus (QTL) mapping, we found 9 QTL for salt tolerance (qST) at the seedling stage located on Chr. 1, 3, 4, 5, 6, 8, and 10. In addition, sequence variant analysis within the QTL regions demonstrated that SKC1/HKT8/HKT1;5 and HAK6 transporters along with numerous transcriptional factors were the candidate genes for the salt tolerant QTL. The DJ15/Koshihikari recombinant inbred lines that contained both qST1.2 and qST6, two QTL with the highest effect for salt tolerance, were more tolerant than the parental lines under salt-stress field conditions. Furthermore, the qST6 near-isogenic lines with IR29 background were more tolerant than IR29, indicating that qST1.2 and qST6 could improve salt tolerance in rice. Overall, our study indicates that wild rice genes could markedly improve the salt tolerance of cultivated rice.

Keywords: wild rice, cultivated rice, salt tolerance, QTL, gene introgression

\section{INTRODUCTION}

Rice is one of the most important staple crops worldwide. The global demand for staple crops, including rice, increases with the continuous increase of human population. However, the production of staple crops is threatened by abiotic stresses including salt, drought and high/low temperature (Arzani and Ashraf, 2016; Calanca, 2017). Therefore, improving the productivity of crops in salt-stressed areas is considered essential to meet the increasing food demand.

Compared with wheat, barley and cotton, rice is a more salt sensitive crop (Chinnusamy et al., 2005). Salt tolerance in rice changes with age; it is salt sensitive at the seedling stage, becomes moderately salt tolerant at the vegetative stage, and highly sensitive at reproductive stage (Lutts et al., 1995; Zeng et al., 2002). Additionally, the salt tolerance is genotype-dependent; i.e., the Oryza 
sativa Indica cultivar Nona Bokra is highly salt tolerant, the $O$. sativa Japonica cultivar Nipponbare is moderately salt tolerant, while the $O$. sativa Japonica cultivar Koshihikari is highly salt sensitive (Kurotani et al., 2015). Salt stress often causes photosynthesis decrease, plant growth inhibition, biomass loss, and partial sterility, all of which lead to yield reduction (Khatun and Flowers, 1995; Pardo, 2010; Munns, 2011; Todaka et al., 2012).

Salt tolerance is a polygenic trait controlled by several quantitative loci (QTL) (Ismail and Horie, 2017).From a mapping population derived from a indica/indica cross, 11 QTL for $\mathrm{Na}^{+}$ uptake, $\mathrm{K}^{+}$uptake, and $\mathrm{Na}^{+} / \mathrm{K}^{+}$selectivity in rice were mapped to regions on chromosomes (Chr.) 1, 4, 6, and 9, and the QTL for $\mathrm{Na}^{+}$and $\mathrm{K}^{+}$uptake were on different linkage groups (Koyama et al., 2001). In a population derived from a cross between Nona Bokra and Koshihikari, 11 QTL for salt tolerance were identified on Chr. 1, 4, 6, 7, and 9, of which two major QTL, qSNC-7 for shoot $\mathrm{Na}^{+}$concentration and qSKC-1 for shoot $\mathrm{K}^{+}$ concentration, explained more than $40 \%$ of the total phenotypic variance (Lin et al., 2004). The major QTL Saltol, for shoot $\mathrm{K}^{+} / \mathrm{Na}^{+}$homeostasis in the salt tolerant cultivar Pokkali on Chr. $1(10.7-12.2 \mathrm{Mb})$, explained $43 \%$ of the variation for seedling shoot $\mathrm{Na}^{+} / \mathrm{K}^{+}$ratio in a RIL population between indica varieties IR29 and Pokkali (Bonilla et al., 2002; Mohammadi-Nejad et al., 2008; Thomson et al., 2010). And 13 QTL for salt tolerance at seedling stage in the salt tolerantcultivar Changbai10 on Chr. 1, 5, 6 , and 7 were identified by linkage mapping, of which 6 QTL were validated by association analysis of 341 japonica rice accessions (Zheng et al., 2015). Although numerous QTL for salt tolerance in rice have been identified, only SKC1, encoding an HKT-type transporter, was cloned by map-based cloning in the qSKC-1 region of Chr. 1 (Lin et al., 2004; Ren et al., 2005). Therefore, further research is needed to characterize genes for salt tolerance in rice.

Wild rice germplasm is considered a valuable source of genes for tolerance to biotic and abiotic stresses, which can be potentially used in rice breeding (Zhang and Xie, 2014; Arzani and Ashraf, 2016; Brozynska et al., 2016; Mishra et al., 2016; Menguer et al., 2017). A previous study identified 13 QTL associated with salt tolerance in the wild rice species $O$. rufipogon Griff. (Tian et al., 2011). Introgression of PcINO1 gene that encodes L-myoinositol 1-phosphate synthase in the wild rice species $O$. coarctata was introgressed into $O$. sativa Indica cultivar and improved its salt tolerance (Das-Chatterjee et al., 2006). OsHKT1 (high-affinity potassium transporter), OsHKT7, as well as numerous transcription factor genes, including zinc finger proteins (ZFPs), NAC (NAM, ATAF, and CUC), MYB, and AP2/ERF (APETALA2/ethylene response factor) are differentially expressed in Dongxiang wild rice under salt stress conditions (Zhou et al., 2016), suggesting that multiple genes are responsible for salt tolerance in wild rice.

From 1970s wild rice genes have been introgressed into cultivated rice, and most of these studies focused on the improvement resistance/tolerance to biotic stresses (Brar and Khush, 1997; Zamir, 2001; Hajjar and Hodgkin, 2007; Dempewolf et al., 2017). Therefore, information regarding the improvement of salt tolerance in cultivated rice using wild rice genes is limited.
We previously identified several genes regulating plant abiotic stress tolerance in Arabidopsis, tobacco and rice (Quan et al., 2007, 2010, 2017; Wan et al., 2011; Zhang et al., 2011). In this study, we isolated a salt tolerant introgression line Dongxiang/Ningjing 15 (DJ15) from the population of the salttolerant wild rice line Dongxiang (O. rufipogon Griff.) (Song et al., 2005; Tian et al., 2011; Zhou et al., 2016) hybridized to a cultivated rice variety (O. sativa ssp. japonica) Ningjing16 (NJ16). Our objectives were to (1) investigate the distribution pattern of genomic fragments introgressed into DJ15, (2) map QTL for salt tolerance, and (3) utilize newly identified QTL in rice breeding for improving salt tolerance.

\section{MATERIALS AND METHODS}

\section{Plant Materials}

Dongxiang wild rice, which was the northernmost population of common wild rice naturally grown in Jiangxi Province, China (N28.14) (Gao et al., 2000; Liu et al., 2017), was crossed to a cultivated japonica rice Ningjing16 (NJ16) four times (Figure 1). The salt tolerance of introgression lines was evaluated against NJ16 and a reference cultivar 96D10, which was widely planted in North China. Based on the method (Figure 1), the salt tolerant introgression line Dongxiang/Ningjing 15 (DJ15) was selected for further study.

\section{Determination of Rice Salt Tolerance}

Two hundred rice seeds of each sample were exposed to $45^{\circ} \mathrm{C}$ for 3 days, then soaked in water containing 0,100 , and $150 \mathrm{mM}$ $\mathrm{NaCl}$ at $25^{\circ} \mathrm{C}$ overnight, and placed at $35^{\circ} \mathrm{C}$ for 3 days. And the germination rate was calculated.

Rice seeds were treated at $45^{\circ} \mathrm{C}$ for 3 days, soaked in water at $25^{\circ} \mathrm{C}$ overnight, and placed at $35^{\circ} \mathrm{C}$ for 3 days. Forty uniformly germinated seeds were sown on metal meshes floating on Yoshida solution (Yoshida et al., 1976) and grown at $26^{\circ} \mathrm{C}$. Seedlings at 10-days stage were transferred to Yoshida solution containing 0 , 100 , and $150 \mathrm{mM} \mathrm{NaCl}$ for comparison among DJ15, NJ16 and 96D10, and $120 \mathrm{mM} \mathrm{NaCl}$ for $\mathrm{F}_{2: 4}$ population. The number of wilted/dead leaves was counted 7 days later (Gao and Lin, 2013).

One-month rice seedlings were transferred to saline field [electrical conductivity of saturated soil extract $(\mathrm{ECe})=5.7$ $\mathrm{dS} \mathrm{m}^{-1}, \mathrm{pH}$ 8.45], and 1-month later the survival rates were checked. When seeds matured, agronomic parameters, including tillering number, plant height and panicle number, were determined, and the yield was calculated after harvest. ECe was determined every week, and controlled to a range of 5.7-5.9 $\mathrm{dS} \mathrm{m} \mathrm{m}^{-1}$ by the irrigation of fresh water or saline water as required.

\section{Analysis of the Distribution of Single Nucleotide Polymorphism (SNP)/ Insertion or Deletion (Indel) Variants}

Genomic resequencing of DJ15 and NJ16 was conducted by a high-throughput sequencing platform (Hiseq2500, Illumina, Inc.; San Diego, CA, U.S.), and the resequencing data were deposited in the National Center for Biotechnology Information Sequence Read Archive (NCBI SRA) under the accession numbers SRR6423773 and SRR6423774. The resequencing data 


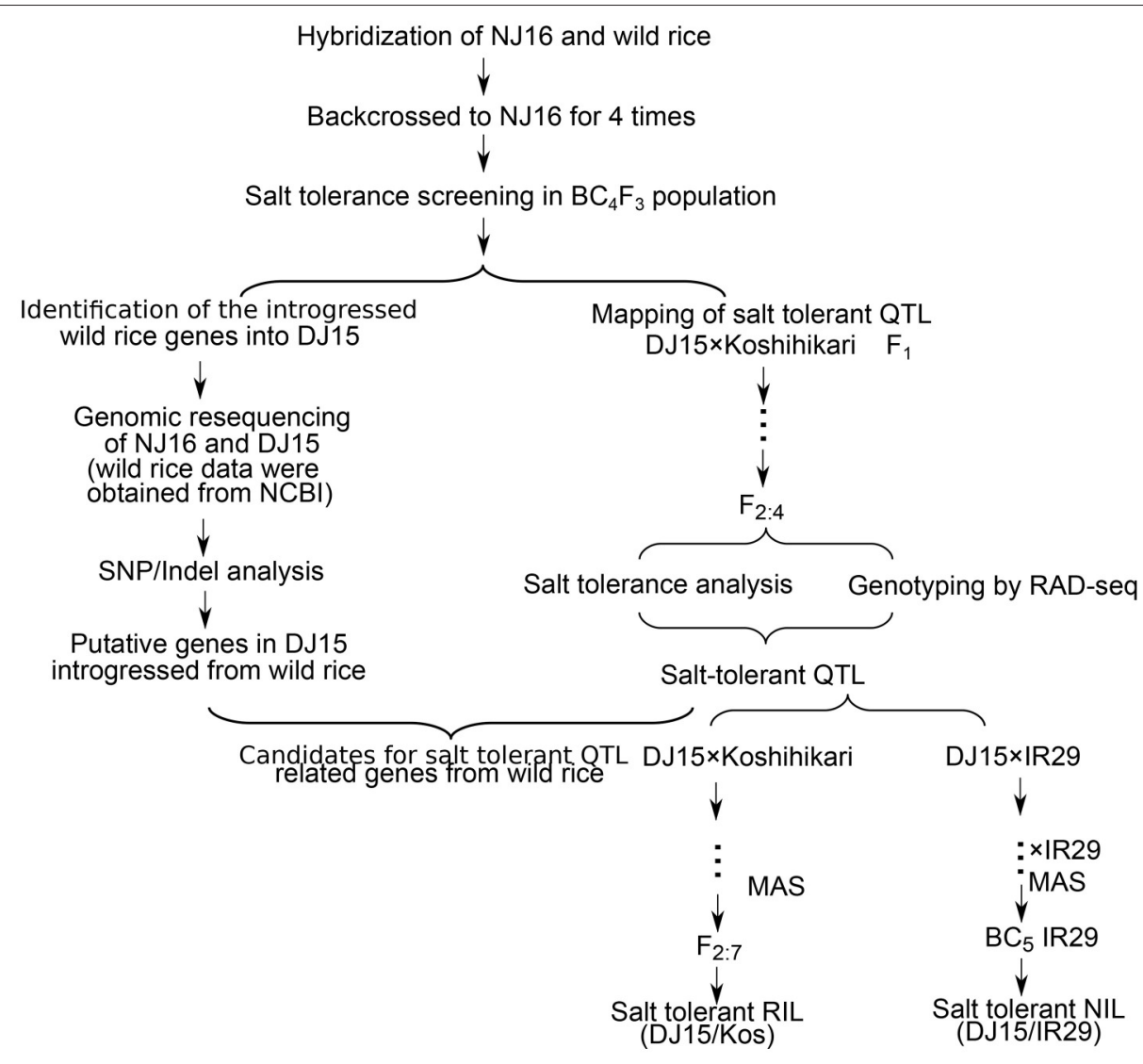

FIGURE 1 | Flowchart that describes the study. Abbreviations: BC, backcross; Kos, Koshihikari; MAS, marker-assisted selection; RAD-seq, restriction-site-associated DNA sequencing.

of Dongxiang wild rice was obtained from NCBI SRA accession SRX158100. The pair-end reads were mapped to Nipponbare rice reference genome (MSU Rice Genome Annotation Project Release 7) (Kawahara et al., 2013) by BWA (Li and Durbin, 2009). Then SNP/Indel variants were called using GATK (McKenna et al., 2010; DePristo et al., 2011). Finally, SNP/Indel density was calculated from the variation data by VCFtools (Danecek et al., 2011).

To verify the Indel variants derived from resequencing analysis, we designed specific PCR primers for randomly selected Indel variants with $3 \mathrm{bp}$ or more difference, spreading over the 12 chromosomes (Table S1). PCR amplification was conducted using genomic DNA isolated from NJ16, DJ15 and Dongxiang wild rice as templates. And the PCR products were separated by electrophoresis in $4 \%$ agarose gel.

\section{Generation of QTL Mapping Population}

DJ15 was crossed with the salt sensitive and late maturing Japonica cultivar Koshihikari. $\mathrm{F}_{3}$ and $\mathrm{F}_{4}$ were obtained by harvesting separately from responding $\mathrm{F}_{2}$ parents, which were named as $\mathrm{F}_{2: 3}$ and $\mathrm{F}_{2: 4}$, respectively.

DJ15 was backcrossed to a salt sensitive indica rice variety IR29 (recurrent parent) five times, along with the confirmation of QTL in the offspring by marker assisted screening at each generation.

\section{Population Genotyping by Restriction-Site-Associated DNA Sequencing (RAD-Seq)}

Genomic DNA was extracted from $103 \mathrm{~F}_{2: 4}$ lines and the two parental lines using the cetyl trimethylammonium bromide (CTAB) method (Murray and Thompson, 1980). Sequencing libraries were constructed from the genomic DNA using the method modified from previous description (Sun et al., 2013). In brief, genomic DNA was incubated at $37^{\circ} \mathrm{C}$ with $\mathrm{Mse}$, T4 DNA ligase, ATP and MseI adapter. After heat-inactivation of the restriction/ligation reactions at $65^{\circ} \mathrm{C}$, the samples were digested with RsaI and Hpy166II, then the subsequent steps for library construction were carried out as detailed in previous description (Sun et al., 2013). Then, pair-end sequencing was performed using a high-throughput sequencing platform (Hiseq2500, Illumina, Inc.; San Diego, CA, U.S.). Finally, specificlocus amplified fragments (SLAFs) were processed to obtain the SLAF genotype of each sample (Sun et al., 2013).

\section{QTL Analysis}

A total of 5,472 polymorphic SLAFs was obtained from the RADseq data of DJ15 and Koshihikari. Using HighMap method as described by Liu et al. (2014), a high density linkage map was constructed from the SLAF genotyping data of $103 \mathrm{~F}_{2: 4}$ lines. 
Next, QTL were identified by the composite interval mapping method using Windows QTL Cartographer (Silva et al., 2012). The LOD threshold of QTL significance was determined by a permutation test $(1,000$ replications $)$ at $P<0.05$.

\section{Identification of Candidate Genes for Salt Tolerance in QTL}

The genomic resequencing data of Koshihikari was obtained from NCBI (DRX002963). After aligning of the pair-end reads to rice reference genome (MSU Rice Genome Annotation Project Release 7) (Kawahara et al., 2013) using BWA (Li and Durbin, 2009), SNP/Indel variants were called using GATK (McKenna et al., 2010; DePristo et al., 2011). The different SNP/Indel variants of DJ15, Koshihikari, NJ16, and Dongxiang were processed with GATK (McKenna et al., 2010; DePristo et al., 2011), and annotated with SnpEff (Cingolani et al., 2012). Candidate genes for salt tolerance in the QTL regions were obtained after filtering out transposons and pseudogenes.

\section{RESULTS}

\section{Evaluation of DJ15 for Salt Tolerance}

The salt-tolerant introgression line DJ15 was selected by lab screening and field evaluating the populations of Dongxiang/Ningjing16 (Ningjing16 as the recurrent parent) at backcross2 $\left(\mathrm{BC}_{2}\right)$ to $\mathrm{BC}_{4}$ generations (Figure 1). Under non-stress conditions, the germination rate of DJ15 was not significantly different from that of the parental variety NJ16 and the control variety 96D10 (Figure 2A). However, at the $150 \mathrm{mM}$ $\mathrm{NaCl}$ treatment, the germination rate of DJ15 was reduced by

\section{A}
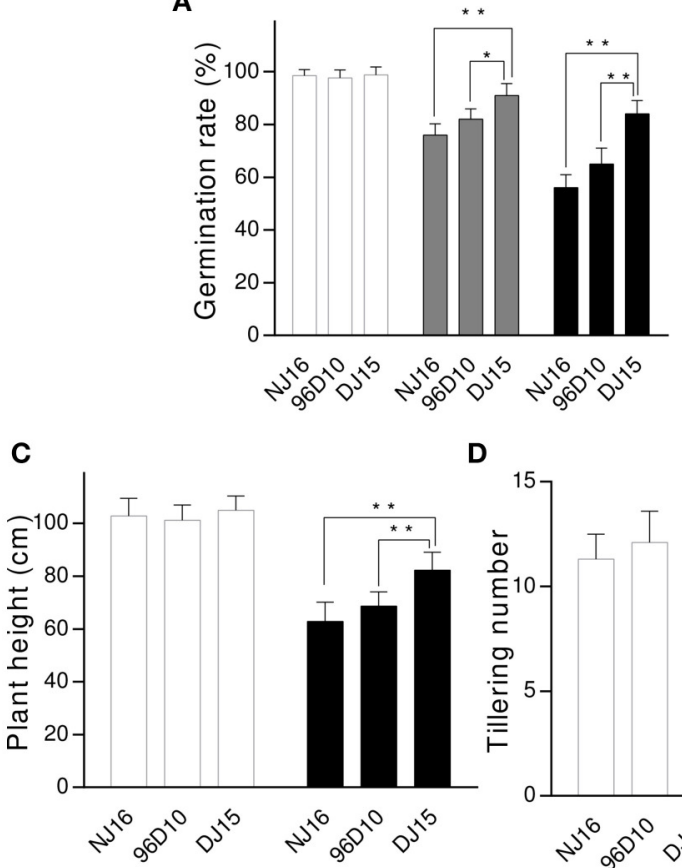

$\mathbf{F}$

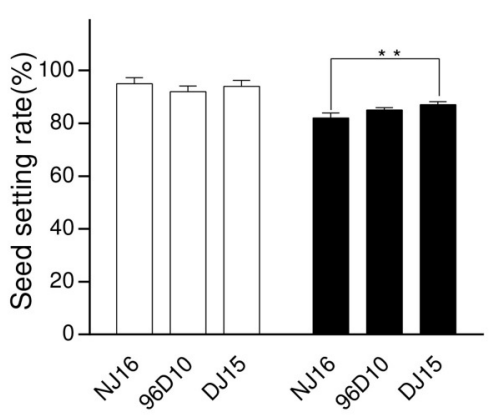

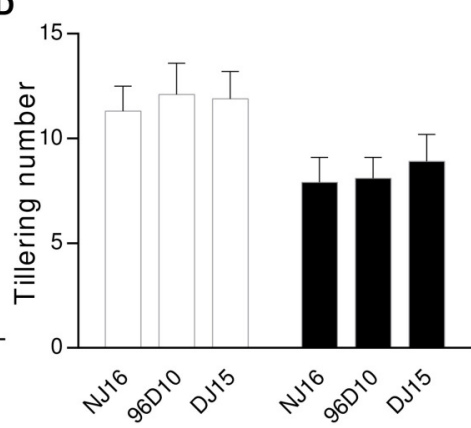

G

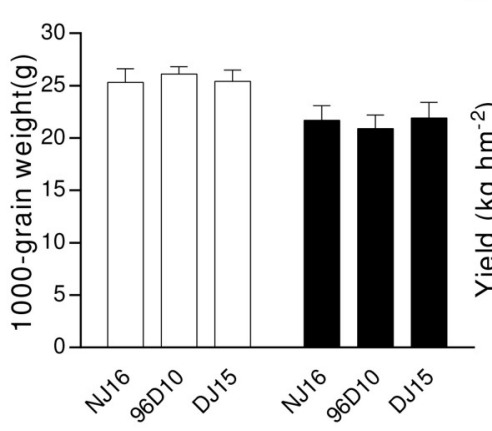

E

H
B

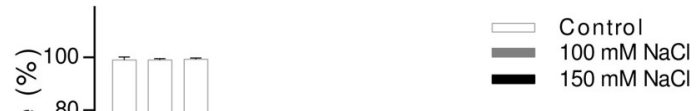

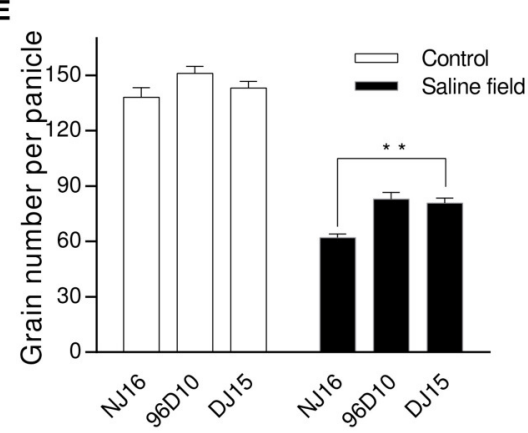

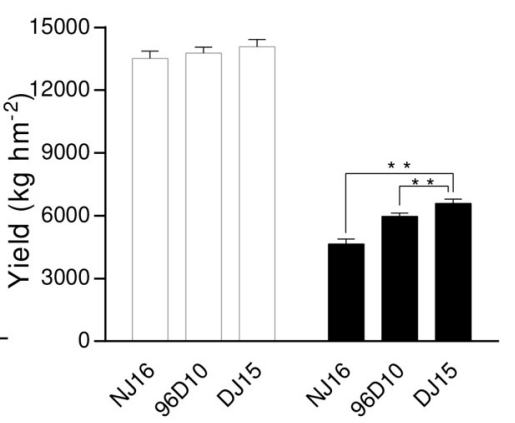


$16 \%$, of NJ16 by $44 \%$ and of $96 \mathrm{D} 10$ by $35 \%$, suggesting that DJ 15 was more salt tolerant than NJ16 and 96D10 at the germination stage.

At the 100 and $150 \mathrm{mM} \mathrm{NaCl}$ treatment, the percentage of wilted/dead leaves was 59 and $42 \%$ for DJ15, 37 and 30\% for NJ16, and 41 and $31 \%$ for 96D10, respectively, indicating that DJ15 was also more salt tolerant than NJ16 and 96D10 at the seedling stage (Figure 2B).

Under non-stress field conditions $\left(\mathrm{ECe}=1.3 \mathrm{dS} \mathrm{m}{ }^{-1}, \mathrm{pH}\right.$ 7.01 ), the yield of DJ15 was slightly higher than that of NJ16 and 96D10; however, plant height, 1,000-grain weight, grain number per panicle, and effective tillering per plant were not significantly different among DJ15, NJ16 and 96D10. Under salt-stress field conditions ( $\left.\mathrm{ECe}=5.7 \mathrm{dS} \mathrm{m}^{-1}, \mathrm{pH} 8.45\right)$, the yield of DJ15 was markedly higher than that of NJ16 and 96D10, possibly due to its higher plant height and effective tillering number, grain number and seed setting rate (Figures $\mathbf{2} \mathbf{C}-\mathbf{H}$ ).

Therefore, these results suggested that DJ15 was more salt tolerant than NJ16 and 96D10 at the germination, seedling and mature stages (Figure 2).

\section{Identification of Introgressed Genomic Fragments in DJ15}

By comparing SNP/Indel variants of DJ15, NJ16 and Dongxiang wild rice, we found 642,349 SNP/Indel variants between DJ15 and NJ16 across the 12 chromosomes, among which 265,862 variants might be obtained from Dongxiang wild rice (Table 1). More than $97 \%$ of $617,227 \mathrm{SNP} /$ Indel variant effects were in intergenic, intron, downstream and upstream of the coding regions, whereas only $2.3 \%$ in exons (Table 2 ).

PCR amplification using specific primers for 52 randomly selected Indel variants showed that different fragments were amplified from the genomic DNA of DJ15, NJ16, and Dongxiang (Figure 3A, Figure S1; Table S1). This result indicated that the identified variants from high-throughput sequencing were accurate.

TABLE 1 | Number of SNP/Indel variants in the O. rufipogon line Dongxiang, the $O$. sativa Japonica variety Ningjing16 ( $\mathrm{NJ16}$ ), and the salt-tolerant introgression line DJ15.

\begin{tabular}{lcc}
\hline Chromosome & DJ15 vs. NJ16 & (DJ15 = Dongxiang) vs. NJ16 \\
\hline 1 & 29,164 & 11,550 \\
2 & 41,189 & 12,497 \\
3 & 14,797 & 6,090 \\
4 & 22,665 & 13,017 \\
5 & 34,779 & 9,396 \\
6 & 156,245 & 85,301 \\
7 & 62,336 & 21,950 \\
8 & 25,905 & 11,802 \\
9 & 18,535 & 9,817 \\
10 & 64,841 & 19,714 \\
11 & 158,873 & 59,294 \\
12 & 13,020 & 5,434 \\
\hline Total & 642,349 & 265,862
\end{tabular}

The rate of variants between NJ16 and Dongxiang wild rice was one variant for every 194 bases, which was markedly higher than that between NJ16 and DJ15 (one variant for every 581 bases). Therefore, the genomic regions introgressed from Dongxiang wild rice into DJ15 were probably enriched with SNP/Indel variants. The regions of high density in SNP/Indel variants between DJ15 and NJ16 (receipt line) distributed unequally over the 12 rice chromosomes, within which four large regions of high density were located in Chr. 6, 7, 10, and 11 (Figure 3B). The distribution pattern of SNPs/Indels was identical between DJ15 and Dongxiang wild rice, different between NJ16 and Dongxiang, and similar between DJ15 and NJ16. These results indicated that the regions of high density in SNP/Indel variants identified in DJ15 might be large DNA fragments introgressed from Dongxiang wild rice.

\section{QTL Mapping}

QTL for salt tolerance were mapped in an $\mathrm{F}_{2: 4}$ population ( $n$ $=103$ ) derived from a cross between DJ15 and a salt sensitive japonica rice variety Koshihikari. Lab and field screening for salt tolerance showed that the survival rate of DJ15 seedlings was $75 \%$, whereas that of Koshihikari was $37 \%$. Curve fitting by Gaussian method showed that the distribution of salt tolerance in $\mathrm{F}_{2: 4}$ lines was close to normal with a slight skewness to the salt sensitivity side, since $61 \%$ of the lines congregated between DJ15 and Koshihikari, $15 \%$ of the lines were more salt sensitive than Koshihikari, and $24 \%$ of the lines were more salt tolerant than DJ15 (Figure 4A).

We used restriction-site-associated DNA sequencing to determine the genotype of DJ15, Koshihikari and the $\mathrm{F}_{2: 4}$ lines. DJ15 and Koshihikari were sequenced at an average depth of $23.98 \times$, whereas $\mathrm{F}_{2: 4}$ lines were sequenced at an average depth of $4.18 \times$. We found a total of 136,724 specific-locus amplified fragments (SLAFs), of which $5.49 \%$ were polymorphic SLAFs (Tables S2, S3).

Mapping SLAFs to the rice reference genome (MSU Rice Genome Annotation Project Release 7, http://rice.plantbiology. msu.edu/) showed that they were nearly equally distributed over

TABLE 2 | Number of SNP/Indel effects by genomic region.

\begin{tabular}{lcc}
\hline Type (alphabetical order) & Count & Percent(\%) \\
\hline Downstream & 138,852 & 22.496 \\
Exon & 14,534 & 2.355 \\
Intergenic & 217,168 & 35.184 \\
Intron & 33,819 & 5.479 \\
Splice_site_acceptor & 39 & 0.006 \\
Splice_site_donor & 32 & 0.005 \\
Splice_site_region & 805 & 0.13 \\
Transcript & 53,643 & 8.691 \\
Upstream & 145,527 & 23.578 \\
Utr_3_prime & 7,861 & 1.274 \\
Utr_5_prime & 4,947 & 0.801 \\
\hline Total & 617,227 & $100 \%$
\end{tabular}


A

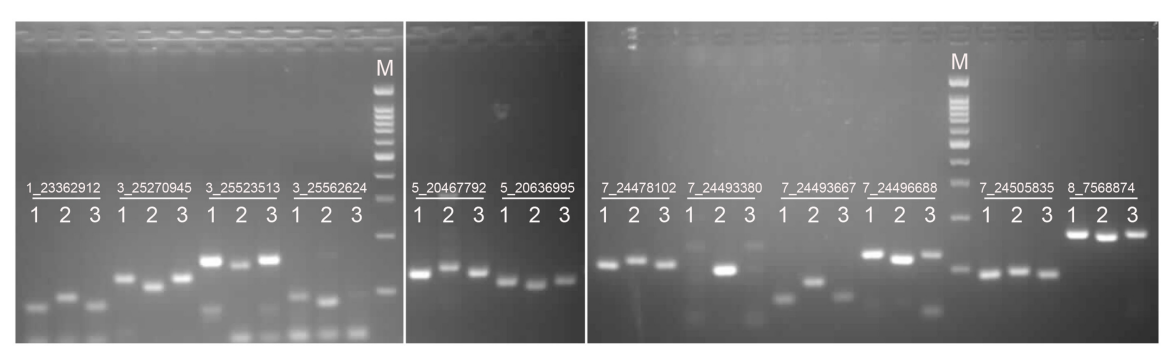

B

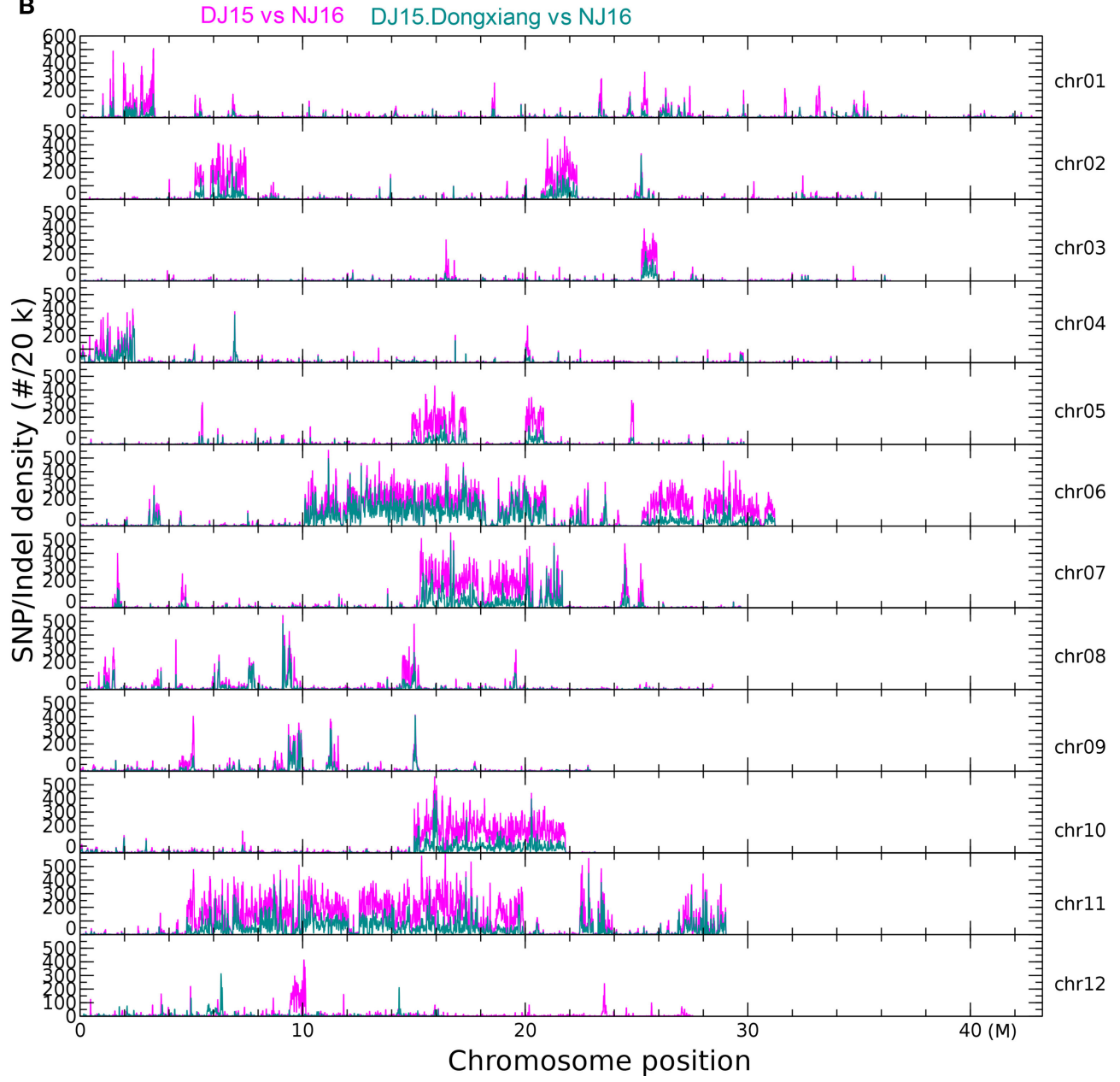

FIGURE 3 | Distribution of single nucleotide polymorphism (SNP)/insertion or deletion (Indel) variants in rice genome. (A) PCR verification of Indels with 3 bp or more difference among the O. rufipogon line Dongxiang (1), the O. sativa Japonica variety Ningjing16 (2), and the salt-tolerant introgression line DJ15 (3). M. 100 bp ladder. (B) Density of SNP/Indel variants in rice genome. "DJ15 vs. NJ16" indicates SNP/Indel variants between DJ15 and NJ16; "DJ15.Dongxiang vs. NJ16" indicates SNP/Indel variants identical between DJ15 and Dongxiang, but different from NJ16.

the 12 rice chromosomes. However, polymorphic SLAFs were unequally distributed over the chromosomes and condensed to six regions of high density on Chr. 2, 5, 6, 7, 10, and 11 (Figures 4B,C).

After filtering SLAFs with sequencing depth under $10 \times$ or heterozygous in parents, we obtained 5,479 polymorphic SLAFs. Then using HighMap software (Liu et al., 2014), we constructed a high density linkage map from SLAF-seq genotyping data of DJ15 $\times$ Koshihikari $\mathrm{F}_{2: 4}$ lines. After filtering makers with MLOD $<5$, we arranged 5,472 of 5,479 SLAF markers to 12 linkage groups, with an average distance of $0.34 \mathrm{cM}$ between two adjacent markers. The linkage map was consistent with the rice reference physical map as revealed by mapping SLAFs to the rice reference genome and finding a Spearman's correlation 


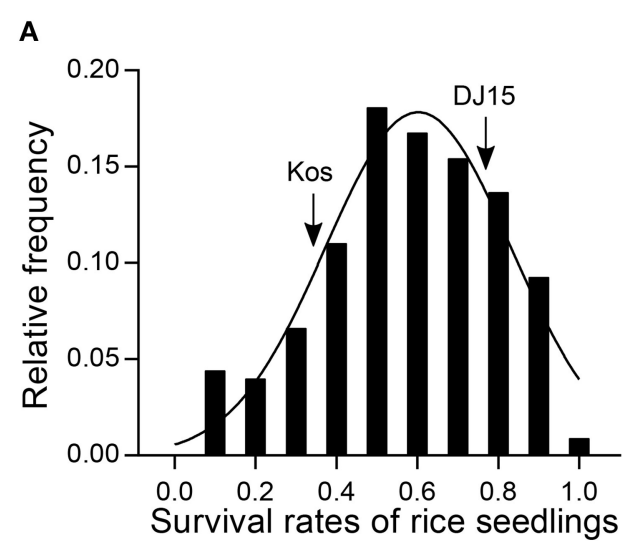

C

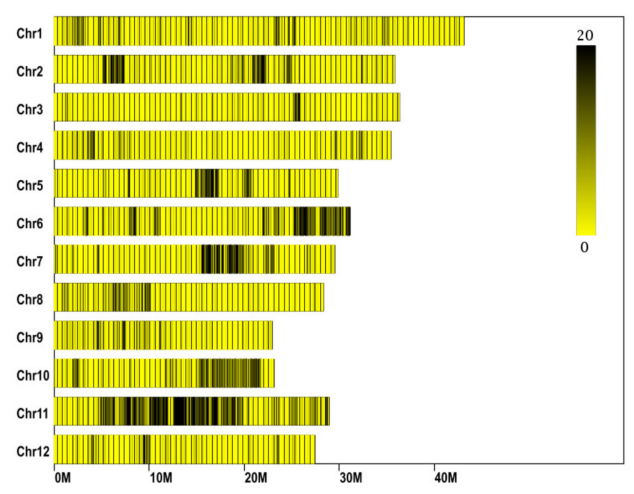

B

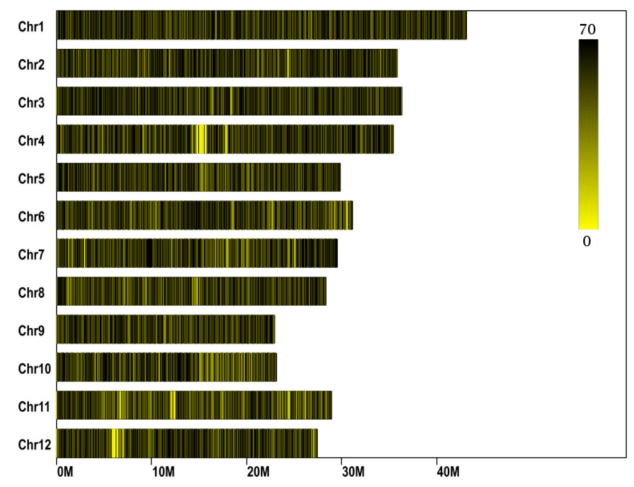

D

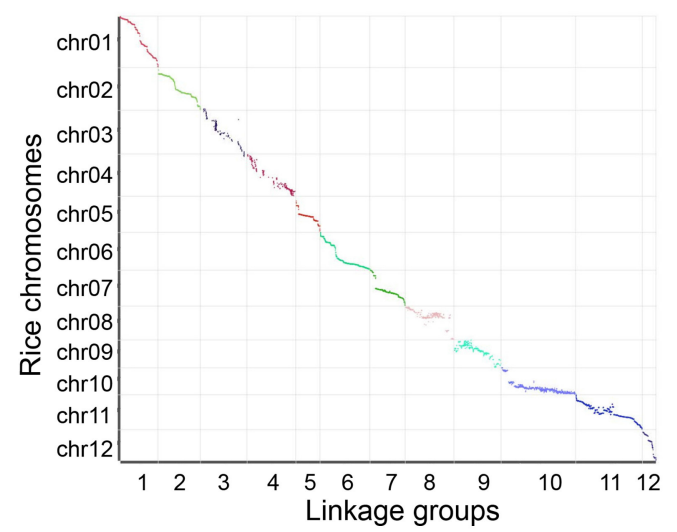

FIGURE 4 | Salt tolerance screening and genotyping of DJ15 $\times$ Koshihikari $F_{2: 4}$ rice population. (A) Survival rate of 2-week rice seedlings after treatment at $120 \mathrm{mM}$ $\mathrm{NaCl}$ for $10 \mathrm{~d}$. Arrows indicate the survival rate of DJ15 and Koshihikari (Kos), respectively. (B) Distribution of specific-locus amplified fragments (SLAFs) in the rice reference genome. (C) Distribution of polymorphic SLAFs between DJ15 and Koshihikari in rice reference genome. Color bars indicate the SLAF number in the rice genome within $1 \mathrm{M}$ window. (D) Comparison of the linkage map constructed from DJ15 $\times$ Koshihikari $\mathrm{F}_{2: 4}$ population with the rice reference physical map.

coefficient of approximately one (Figure 4D). Thus, the marker positions on the linkage map were valid and the genotyping results were accurate.

Using composite interval mapping, we found 9 QTL for salt tolerance spreading over 7 chromosomes with an LOD cutoff $>$ 3.0 (Table 3). The positive additive effects of 7 QTL were derived from DJ15 alleles, and the total phenotypic variance explained by each QTL was 8.81-22.10\%. Of these, qST6 accounted for $19.38 \%$ of the total phenotypic variance, and had an additive effect of 0.12 for increasing survival rate derived from DJ15 allele.

\section{Candidate Genes for Salt Tolerance in QTL}

To identify candidate genes for salt tolerance in the QTL regions, we analyzed the sequence variants of NJ16, DJ15, Koshihikari and Dongxiang wild rice in the 9 QTL regions (Table S4). Among the candidate genes, we found Os01g0307500 (OsSKC1) located in the qST1.2 region and Os01g0932500 (OsHAK6) located in the qST1.1 region. Other QTL candidates included several transcription factors (MYB, zinc finger and AP2/ERF) and protein kinases, which might stimulate plant salt stress tolerance by transcriptional regulation and kinase pathways (Golldack
TABLE 3 | QTL for salt tolerance identified in DJ15 × Koshihikari F 2:4 segregating population.

\begin{tabular}{lccccc}
\hline QTL & Chromosome & $\begin{array}{c}\text { Chromosome } \\
\text { position (M) }\end{array}$ & Peak LOD & $\begin{array}{c}\text { Additive } \\
\text { effect }^{\mathbf{a}}\end{array}$ & $\mathbf{R}^{\mathbf{2}}(\%)^{\mathbf{b}}$ \\
\hline qST1.1 & 1 & 40.3 & 4.90 & 0.017 & 22.10 \\
qST1.2 & 1 & 10.6 & 3.30 & 0.038 & 10.00 \\
qST3 & 3 & 6.2 & 3.61 & 0.022 & 14.27 \\
qST4.1 & 4 & 29.6 & 3.65 & 0.15 & 9.79 \\
qST4.2 & 4 & 23.4 & 3.55 & -0.096 & 8.81 \\
qST5 & 5 & 16.5 & 3.98 & -0.11 & 12.67 \\
qST6 & 6 & 25.6 & 4.12 & 0.12 & 19.38 \\
qST8 & 8 & 21.7 & 4.33 & 0.019 & 15.80 \\
qST10 & 10 & 17.2 & 3.68 & 0.13 & 11.35 \\
\hline
\end{tabular}

${ }^{a}$ Additive effect on the DJ15 allele.

${ }^{b}$ Percentage of total phenotypic variance explained by the QTL.

et al., 2014). These results indicated that the identified QTL might enhance rice salt tolerance via ionic homeostasis maintenance, gene transcription and kinase signaling pathways. 
TABLE 4 | Agronomic traits of DJ15/Koshihikari recombinant inbred lines (RILs) carrying qST6 ${ }^{\mathrm{DJ} 15}$ under salt-stress field conditions ( $\mathrm{ECe}=4.0 \mathrm{dS} \mathrm{m} \mathrm{m}^{-1}, \mathrm{pH}$ 8.5).

\begin{tabular}{|c|c|c|c|c|c|}
\hline RIL & Tillering No. & Panicle No. & $\begin{array}{c}\text { Plant } \\
\text { height }(\mathbf{c m})\end{array}$ & $\begin{array}{l}\text { Heading } \\
\text { date }\end{array}$ & $\begin{array}{c}\text { Yield } \\
\left(\mathrm{kg} \mathrm{hm}^{-2}\right)\end{array}$ \\
\hline YD7 & 10.2 & 9.2 & 94.7 & Aug 1 & 11,743 \\
\hline YD8 & 9.2 & 7.8 & 93.6 & Aug 2 & 11,411 \\
\hline YD22 & 13.0 & 11.0 & 97.2 & Aug 5 & 10,085 \\
\hline YD23 & 12.0 & 11.0 & 94.0 & Aug 4 & 10,275 \\
\hline YD28 & 14.4 & 12.2 & 98.4 & Aug 8 & 10,511 \\
\hline YD44 & 10.0 & 9.4 & 97.3 & Aug 2 & 10,606 \\
\hline DJ15 & 13.8 & 11.6 & 88.7 & Jul 28 & 9,521 \\
\hline Koshihikari & 16.4 & 14.4 & 94.3 & Aug 9 & 7,386 \\
\hline
\end{tabular}

\section{Application of QTL for Improvement of Salt Tolerance in Cultivated Rice}

As DJ15 is a salt tolerant and early maturing introgression line which is only suitable for Ningxia but not suitable for planting in other areas of Northeast China, we investigated if it would be possible to identify a highly salt-tolerant and late maturing line by hybridization of DJ15 to a salt sensitive but late maturing rice variety Koshihikari. By marker-assisted screening, we identified 55 lines carrying qST1.2 $2^{\text {DJ15 }}$ and qST6 ${ }^{\mathrm{DJ15}}$ from 980 DJ15/Koshihikari recombinant inbred lines (RILs). Under saltstress field conditions $\left(\mathrm{ECe}=4.0 \mathrm{dS} \mathrm{m}^{-1}\right.$ ), the heading dates of 6 (YD7, 8, 22, 23, 28, 44) of 55 lines were about $4-11$ days later than that of DJ15, and their yields were $8-23 \%$ more than that of DJ 15 or $36-59 \%$ more than that of Koshihikari (Table 4). Therefore, the 6 salt tolerant, high yielding and late maturing lines might be used for developing new rice varieties.

qST1.2 and qST6 were two QTL that showed the highest effect for salt tolerance in DJ15, and SKC1 might be one major effect gene for qST1.2 (Ren et al., 2005). Among the 9 newly identified QTL for salt tolerance, qST6 was a novel QTL in a region of high SNP/Indel variant density; thus, we backcrossed DJ15 to IR29 5 times and screened the offspring by qST6 linked markers to select IR29 near-isogenic line (NIL) ${ }^{\mathrm{D} 115}$. Under non-stress conditions, no significant differences were found in the seedling growth of IR29 and IR29 NIL ${ }^{\mathrm{DJ} 15}$ (Figure 5). Lab screening under salt stress conditions $(80 \mathrm{mM} \mathrm{NaCl})$ for $10 \mathrm{~d}$, IR29 $\mathrm{NIL}^{\text {DJ15 }}$ seedlings demonstrated higher survival rate, plant height, and biomass compared with IR29, indicating that IR29 NIL ${ }^{\text {DJ15 }}$ was more tolerant to salt stress (Figure 5). These results suggested that qST1.2 and qST6 could be utilized in rice breeding for improving salt tolerance.

\section{DISCUSSION}

$O$. sativa and O. glaberrima are the only two domesticated species within the genus Oryza, and thus the genetic diversity of cultivated rice is limited. Oryza contains more than 20 wild species, which are considered a significant source of genetic diversity (Atwell et al., 2014) since they compose a valuable pool of genes for disease or abiotic stress resistance/tolerance
(Brar and Khush, 1997; Zamir, 2001; Hajjar and Hodgkin, 2007; Maxted et al., 2012; Mishra et al., 2016; Dempewolf et al., 2017; Wang et al., 2017). Pest/disease resistance is the most well studied trait that can be improved by introgression of wild rice genes (Hajjar and Hodgkin, 2007). In addition, the genome of wild rice contains tolerant alleles of abiotic stress genes. For example, submerge tolerant $O$. rufipogon and $O$. nivara accessions carry the SUB1A-1 allele for submergence tolerance (Niroula et al., 2012). An accession of wild rice (O. rufipogon) from Yunnan Province of China contains 13 QTL for salt tolerance QTL (Tian et al., 2011). Dongxiang wild rice changes the expression of more than 6,800 genes related to salt stress, including numerous transcription factor genes, such as ZFP, NAC, MYB, and ERF, indicating that these transcription factors might regulate multiple regulatory pathways under salt stress conditions (Zhou et al., 2016). However, information regarding the improvement of salt tolerance in cultivated rice using wild genes is limited. In this study, we demonstrate that wild rice genes could enhance the salt tolerance of cultivate rice.

NJ16 is a commercial variety widely cultivated in the Yellowriver irrigating region of Ningxia Province in China. However, the yield is negatively affected by salt stress due to the widespread saline soil in this region. In the present study, we introgressed wild rice fragments into a rice cultivar, and obtained an introgression line with improved salt tolerance after several rounds of selection. These results demonstrated that wild rice genes could be used to improve salt tolerance in cultivated rice.

AtHKT1 promotes $\mathrm{Na}^{+}$unloading from the xylem and increases salt tolerance in Arabidopsis (Davenport et al., 2007; Møller et al., 2009). OsSKC1 (Ren et al., 2005) is a HKT-like protein, namely HKT8/HKT1; (Garciadeblás et al., 2003; Hauser and Horie, 2010). OsSKC1 is not primarily a $\mathrm{K}^{+}$transporter but is a $\mathrm{Na}^{+}$selective transporter, which regulates $\mathrm{Na}^{+} / \mathrm{K}^{+}$levels in shoots by recirculating $\mathrm{Na}^{+}$from shoots to roots, thus affecting the shoot $\mathrm{K}^{+}$content indirectly (Ren et al., 2005). Furthermore, Immuno-staining and ${ }^{22} \mathrm{Na}^{+}$tracer experiments suggested that OsHKT1;5 protein contributes to $\mathrm{Na}^{+}$exclusion in the phloem, in addition to xylem $\mathrm{Na}^{+}$unloading, to prevent $\mathrm{Na}^{+}$transfer to young leaf blades (Kobayashi et al., 2017). $\mathrm{K}^{+}$transporter OsHAK1 mediates $\mathrm{K}^{+}$uptake in roots and $\mathrm{K}^{+}$translocation to shoots, which could enhance salt tolerance (Bañuelos et al., 2002; Chen et al., 2015). Similarly, OsHAK5 enables $\mathrm{K}^{+}$uptake by roots under external $\mathrm{K}^{+}$nutrient limitation and saline conditions (Yang et al., 2014). Therefore, OsHAK6 in qST1.1 might mediate salt tolerance as the OsHAKs mentioned above.

Salt tolerance is a complex trait controlled by many genetic loci. For example, eleven QTL related to salt tolerance are located on 5 chromosomes from the $\mathrm{F}_{3}$ population of Nona Bokra and Koshihikari (Ren et al., 2005). And 13 of 15 QTL on six chromosomes from wild rice improve salt tolerance in Teqing background (Tian et al., 2011). Compared with salt tolerance QTL identified from wild rice O. rufipogon (Tian et al., 2011), in this study the position of qST1.2 is close to qSTS1, and the other 5 QTL for salt tolerance are novel. Up to now, only SKC1 localized in qST1.2 region has been cloned by mapping (Ren et al., 2005). Therefore, the function of other candidate genes for salt tolerant QTL remains to be explored in future studies. 

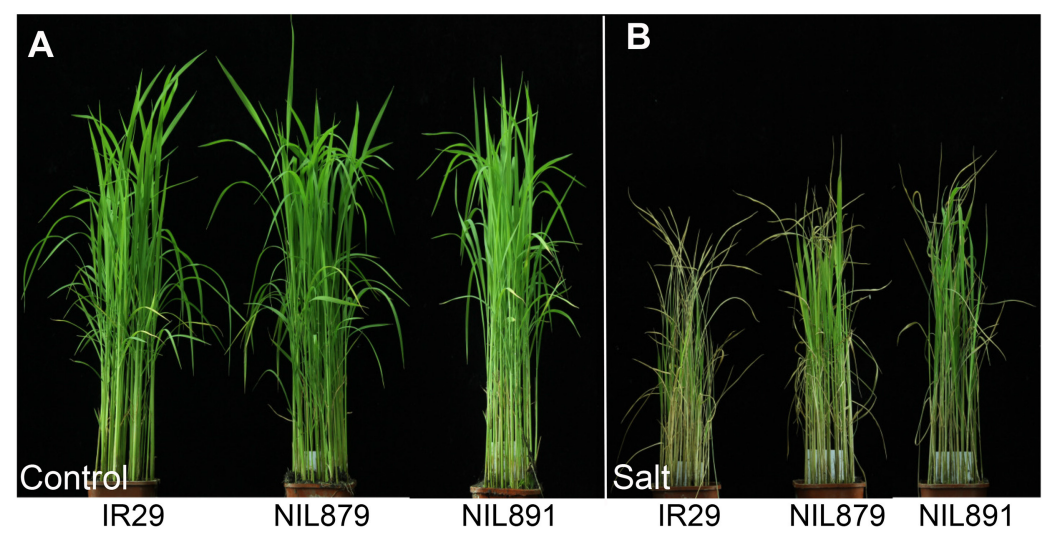

C

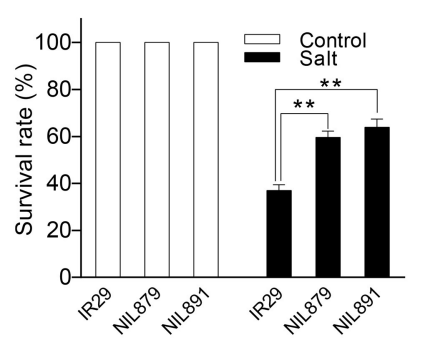

D

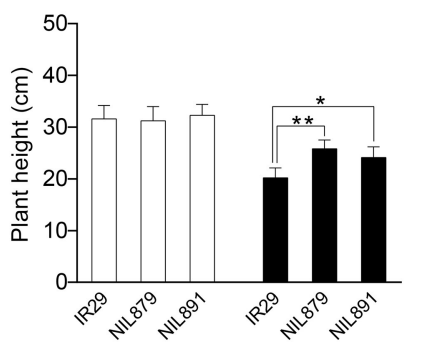

$E$

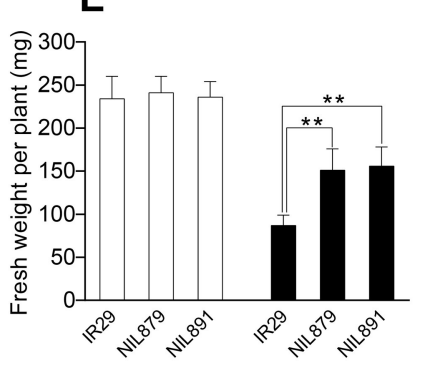

FIGURE 5 | Salt tolerance of qST6 ${ }^{\text {DJ15 }}$ near isogenic lines. (A-E) Survival rate, plant height, and fresh weight of 2-week-old IR29 and qST6 DJ15 NILs (NIL879 and NIL891) seedlings after treatment at $80 \mathrm{mM} \mathrm{NaCl}$ for 10 days and subsequent recovery for 5 days. *Indicates significant difference at $P<0.05$ in Tukey's multiple comparisons test after ANOVA; ${ }^{\star \star}$ Indicates significantly difference at $P<0.01$ in Tukey's multiple comparisons test after ANOVA.

Although several QTL for salt tolerance have been identified from wild rice (Tian et al., 2011), their physiological function has not been confirmed under salt-stress field conditions. In the present study, we obtained 6 salt-tolerant DJ15/Koshihikari RILs, carrying both qST $1.2^{\mathrm{DJ} 15}$ and $\mathrm{qST} 6^{\mathrm{DJ} 15}$, via marker-assisted screening. These RILs showed a lower tillering and panicle number than Koshihikari and DJ15, but a higher yield, under salt-stress field conditions, which might result from the relatively higher seed setting rate of RILs. Additionally, IR29 NIL ${ }^{D J 15}$ was more salt tolerant than IR29 at the seedling stage and showed higher yield per plant in a preliminary study. However, more research is needed to elucidate the underlying regulating mechanism of yield in relation to $\mathrm{qST}^{\mathrm{DJ15}}$ under salt stress conditions.

As DJ15 and the salt tolerant RILs demonstrate variation in growth duration, the growth adaptability in saline field of Dongying (Shandong Province, E118.7, N37.6) and Panjin (Liaoning Province, E122.0, N41.0), other than Yinchuan (Ningxia Province, E106.2, N38.5) in North China, is under investigation. And we hope to release these salt tolerant lines to the public in the near future. Overall, our results demonstrated that wild rice genes could be successfully used in rice breeding programs to develop varieties with markedly improved salt tolerance.

\section{AUTHOR CONTRIBUTIONS}

$\mathrm{RH}$ conceived the project. SL, JH, HB, XL, and YZ constructed wild rice introgression lines to isolate DJ15, and performed saline field tests. JW performed salt tolerance determination by hydroponics in $\mathrm{F}_{2: 4}$ population. RQ performed the rest of experiments and analyzed data. HZ and $\mathrm{ZZ}$ analyzed the data. RQ wrote the manuscript.

\section{ACKNOWLEDGMENTS}

This work was supported by the National Key Research and Development Program of China (2016YFD0100604), National Natural Science Foundation of China (31771706), Ministry of Agriculture of China (2016ZX08009-003-005), and Ningxia Hui Autonomous Region of China (2013NYY Z0302).

\section{SUPPLEMENTARY MATERIAL}

The Supplementary Material for this article can be found online at: https://www.frontiersin.org/articles/10.3389/fpls.2017. 02269/full\#supplementary-material 


\section{REFERENCES}

Arzani, A., and Ashraf, M. (2016). Smart engineering of genetic resources for enhanced salinity tolerance in crop plants. Crit. Rev. Plant Sci. 35, 146-189. doi: 10.1080/07352689.2016.1245056

Atwell, B. J., Wang, H., and Scafaro, A. P. (2014). Could abiotic stress tolerance in wild relatives of rice be used to improve Oryza sativa? Plant Sci. 215-216, 48-58. doi: 10.1016/j.plantsci.2013.10.007

Bañuelos, M. A., Garciadeblas, B., Cubero, B., and Rodríguez-Navarro, A. (2002). Inventory and functional characterization of the HAK potassium transporters of rice. Plant Physiol. 130, 784-795. doi: 10.1104/pp.007781

Bonilla, P., Dvorak, J., Mackill, D., Deal, K., and Gregorio, G. (2002). RFLP and SSLP mapping of salinity tolerance genes in chromosome 1 of rice (Oryza sativa L.) using recombinant inbred lines. Philipp. Agric. Sci. 85, 68-76.

Brar, D., and Khush, G. (1997). Alien introgression in rice. Plant Mol. Biol. 35, 35-47. doi: 10.1023/A:1005825519998

Brozynska, M., Furtado, A., and Henry, R. J. (2016). Genomics of crop wild relatives: expanding the gene pool for crop improvement. Plant Biotechnol. J. 14, 1070-1085. doi: 10.1111/pbi. 12454

Calanca, P. P. (2017). "Effects of abiotic stress in crop production," in Quantication of Climate Variability, Adaptation and Mitigation for Agricultural Sustainability, eds M. Ahmed and C. O. Stockle (Cham: Springer International Publishing), 165-180. doi: 10.1007/978-3-319-32059-5_8

Chen, G., Hu, Q., Luo, L., Yang, T., Zhang, S., Hu, Y., et al. (2015). Rice potassium transporter OsHAK1 is essential for maintaining potassium-mediated growth and functions in salt tolerance over low and high potassium concentration ranges. Plant Cell Environ. 38, 2747-2765. doi: 10.1111/pce.12585

Chinnusamy, V., Jagendorf, A., and Zhu, J.-K. (2005). Understanding and improving salt tolerance in plants. Crop Sci. 45, 437-448. doi: $10.2135 /$ cropsci2005.0437

Cingolani, P., Platts, A., Wang, L. L., Coon, M., Nguyen, T., Wang, L., et al. (2012). A program for annotating and predicting the effects of single nucleotide polymorphisms, SnpEff: SNPs in the genome of Drosophila melanogaster strain $w^{1118}$; iso-2; iso-3. Fly 6, 80-92. doi: 10.4161/fly.19695

Danecek, P., Auton, A., Abecasis, G., Albers, C. A., Banks, E., DePristo, M. A., et al. (2011). The variant call format and VCFtools. Bioinformatics 27, 2156-2158. doi: 10.1093/bioinformatics/btr330

Das-Chatterjee, A., Goswami, L., Maitra, S., Dastidar, K. G., Ray, S., and Majumder, A. L. (2006). Introgression of a novel salt-tolerant L-myo-inositol 1-phosphate synthase from Porteresia coarctata (Roxb.) Tateoka (PcINO1) confers salt tolerance to evolutionary diverse organisms. FEBS Lett. 580, 3980-3988. doi: 10.1016/j.febslet.2006.06.033

Davenport, R. J., Muñoz-Mayor, A., Jha, D., Essah, P. A., Rus, A., and Tester, M. (2007). The $\mathrm{Na}^{+}$transporter AtHKT1;1 controls retrieval of $\mathrm{Na}^{+}$from the xylem in Arabidopsis. Plant Cell Environ. 30, 497-507. doi: 10.1111/j.1365-3040.2007.01637.x

Dempewolf, H., Baute, G., Anderson, J., Kilian, B., Smith, C., and Guarino, L. (2017). Past and future use of wild relatives in crop breeding. Crop Sci. 57:1070. doi: 10.2135/cropsci2016.10.0885

DePristo, M. A., Banks, E., Poplin, R., Garimella, K. V., Maguire, J. R., Hartl, C., et al. (2011). A framework for variation discovery and genotyping using nextgeneration DNA sequencing data. Nat. Genet. 43, 491-498. doi: 10.1038/ng.806

Gao, J.-P., and Lin, H.-X. (2013). "QTL analysis and map-based cloning of salt tolerance gene in rice," in Rice Protocols, Methods in Molecular Biology, Vol. 956, ed Y. Yang (Totowa, NJ: Humana Press), 69-82. doi: 10.1007/978-1-62703-194-3_6

Gao, L., Chen, W., Jiang, W., Song, G. E., Hong, D., and Wang, X. (2000). Genetic erosion in northern marginal population of the common wild rice Oryza rufipogon Griff. and its conservation, revealed by the change of population genetic structure. Hereditas 133, 47-53. doi: 10.1111/j.1601-5223.2000. 00047.x

Garciadeblás, B., Senn, M. E., Bañuelos, M. A., and Rodríguez-Navarro, A. (2003). Sodium transport and HKT transporters: the rice model. Plant J. 34, 788-801. doi: 10.1046/j.1365-313X.2003.01764.x

Golldack, D., Li, C., Mohan, H., and Probst, N. (2014). Tolerance to drought and salt stress in plants: unraveling the signaling networks. Front. Plant Sci. 5:151. doi: $10.3389 /$ fpls.2014.00151
Hajjar, R., and Hodgkin, T. (2007). The use of wild relatives in crop improvement: a survey of developments over the last 20 years. Euphytica 156, 1-13. doi: 10.1007/s10681-007-9363-0

Hauser, F., and Horie, T. (2010). A conserved primary salt tolerance mechanism mediated by HKT transporters: a mechanism for sodium exclusion and maintenance of high $\mathrm{K}^{+} / \mathrm{Na}^{+}$ratio in leaves during salinity stress. Plant Cell Environ. 33, 552-565. doi: 10.1111/j.1365-3040.2009.02056.x

Ismail, A. M., and Horie, T. (2017). Genomics, physiology, and molecular breeding approaches for improving salt tolerance. Annu. Rev. Plant Biol. 68, 405-434. doi: 10.1146/annurev-arplant-042916-040936

Kawahara, Y., de la Bastide, M., Hamilton, J. P., Kanamori, H., McCombie, W. R., Ouyang, S., et al. (2013). Improvement of the Oryza sativa Nipponbare reference genome using next generation sequence and optical map data. Rice 6:4. doi: 10.1186/1939-8433-6-4

Khatun, S., and Flowers, T. J. (1995). Effects of salinity on seed set in rice. Plant Cell Environ. 18, 61-67. doi: 10.1111/j.1365-3040.1995.tb00544.x

Kobayashi, N. I., Yamaji, N., Yamamoto, H., Okubo, K., Ueno, H., Costa, A., et al. (2017). OsHKT1;5 mediates $\mathrm{Na}^{+}$exclusion in the vasculature to protect leaf blades and reproductive tissues from salt toxicity in rice. Plant J. 91, 657-670. doi: 10.1111/tpj.13595

Koyama, M. L., Levesley, A., Koebner, R. M., Flowers, T. J., and Yeo, A. R. (2001). Quantitative trait loci for component physiological traits determining salt tolerance in rice. Plant Physiol. 125, 406-422. doi: 10.1104/pp.125.1.406

Kurotani, K., Yamanaka, K., Toda, Y., Ogawa, D., Tanaka, M., Kozawa, H., et al. (2015). Stress tolerance profiling of a collection of extant salt-tolerant rice varieties and transgenic plants overexpressing abiotic stress tolerance genes. Plant Cell Physiol. 56, 1867-1876. doi: 10.1093/pcp/pcv106

Li, H., and Durbin, R. (2009). Fast and accurate short read alignment with Burrows-Wheeler transform. Bioinformatics 25, 1754-1760. doi: 10.1093/bioinformatics/btp324

Lin, H. X., Zhu, M. Z., Yano, M., Gao, J. P., Liang, Z. W., Su, W. A., et al. (2004). QTLs for $\mathrm{Na}^{+}$and $\mathrm{K}^{+}$uptake of the shoots and roots controlling rice salt tolerance. Theor. Appl. Genet. 108, 253-260. doi: 10.1007/s00122-0031421-y

Liu, D., Ma, C., Hong, W., Huang, L., Liu, M., Liu, H., et al. (2014). Construction and analysis of high-density linkage map using high-throughput sequencing data. PLoS ONE 9:e98855. doi: 10.1371/journal.pone.0098855

Liu, S., Zheng, X., Yu, L., Feng, L., Wang, J., Gong, T., et al. (2017). Comparison of the genetic structure between in situ and ex situ populations of Dongxiang wild rice (Oryza rufipogon Griff.). Crop Sci. 57:3075. doi: 10.2135/cropsci2017.01.0015

Lutts, S., Kinet, J., and Bouharmont, J. (1995). Changes in plant response to $\mathrm{NaCl}$ during development of rice (Oryza sativa L.) varieties differing in salinity resistance. J. Exp. Bot. 46, 1843-1852. doi: 10.1093/jxb/46.12.1843

Maxted, N., Kell, S., Ford-Lloyd, B., Dulloo, E., and Toledo, A. (2012). Toward the systematic conservation of global crop wild relative diversity. Crop Sci. 52, 774-785. doi: 10.2135/cropsci2011.08.0415

McKenna, A., Hanna, M., Banks, E., Sivachenko, A., Cibulskis, K., Kernytsky, A., et al. (2010). The Genome Analysis Toolkit: a MapReduce framework for analyzing next-generation DNA sequencing data. Genome Res. 20, 1297-1303. doi: $10.1101 /$ gr.107524.110

Menguer, P. K., Sperotto, R. A., and Ricachenevsky, F. K. (2017). A walk on the wild side: Oryza species as source for rice abiotic stress tolerance. Genet. Mol. Biol. 40, 238-252. doi: 10.1590/1678-4685-gmb-2016-0093

Mishra, S., Singh, B., Misra, P., Rai, V., and Singh, N. K. (2016). Haplotype distribution and association of candidate genes with salt tolerance in Indian wild rice germplasm. Plant Cell Rep. 35, 2295-2308. doi: 10.1007/s00299-016-2035-6

Møller, I. S., Gilliham, M., Jha, D., Mayo, G. M., Roy, S. J., Coates, J. C., et al. (2009). Shoot $\mathrm{Na}^{+}$exclusion and increased salinity tolerance engineered by cell typespecific alteration of $\mathrm{Na}^{+}$transport in Arabidopsis. Plant Cell 21, 2163-2178. doi: $10.1105 /$ tpc. 108.064568

Mohammadi-Nejad, G., Arzani, A., Rezai, A., Singh, R., and Gregorio, G. (2008). Assessment of rice genotypes for salt tolerance using microsatellite markers associated with the saltol QTL. Afr. J. Biotechnol. 7, 730-36.

Munns, R. (2011). "Plant adaptations to salt and water stress: differences and commonalities," in Plant Responses to Drought and Salinity Stress Developments 
in a Post-genomic Era, Vol 57 of Advances in Botanical Research, ed I. Turkan (London: Academic Press), 1-32. doi: 10.1016/B978-0-12-387692-8.00001-1

Murray, M. G., and Thompson, W. F. (1980). Rapid isolation of high molecular weight plant DNA. Nucleic Acids Res. 8, 4321-4325. doi: 10.1093/nar/8.19.4321

Niroula, R. K., Pucciariello, C., Ho, V. T., Novi, G., Fukao, T., and Perata, P. (2012). SUB1A-dependent and -independent mechanisms are involved in the flooding tolerance of wild rice species. Plant J. 72, 282-293. doi: 10.1111/j.1365-313X.2012.05078.x

Pardo, J. M. (2010). Biotechnology of water and salinity stress tolerance. Curr. Opin. Biotechnol. 21, 185-196. doi: 10.1016/j.copbio.2010.02.005

Quan, R., Hu, S., Zhang, Z., Zhang, H., Zhang, Z., and Huang, R. (2010). Overexpression of an ERF transcription factor TSRF1 improves rice drought tolerance. Plant Biotechnol. J. 8, 476-488. doi: 10.1111/j.1467-7652.2009.00492.x

Quan, R., Lin, H., Mendoza, I., Zhang, Y., Cao, W., Yang, Y., et al. (2007). SCaBP8/CBL10, a putative calcium sensor, interacts with the protein kinase SOS2 to protect Arabidopsis shoots from salt stress. Plant Cell 19, 1415-1431. doi: $10.1105 /$ tpc. 106.042291

Quan, R., Wang, J., Yang, D., Zhang, H., Zhang, Z., and Huang, R. (2017). EIN3 and SOS2 synergistically modulate plant salt tolerance. Sci. Rep. 7:44637. doi: $10.1038 /$ srep44637

Ren, Z. H., Gao, J. P., Li, L. G., Cai, X. L., Huang, W., Chao, D. Y., et al. (2005). A rice quantitative trait locus for salt tolerance encodes a sodium transporter. Nat. Genet. 37, 1141-1146. doi: 10.1038/ng1643

Silva, L. D. C. E., Wang, S., and Zeng, Z.-B. (2012). “Composite interval mapping and multiple interval mapping: procedures and guidelines for using Windows QTL Cartographer," in Quantitative Trait Loci (QTL): Methods and Protocols, Methods in Molecular Biology, Vol. 871, ed Y. Yang (New York, NY: Humana Press), 75-119. doi: 10.1007/978-1-61779-785-9_6

Song, Z., Li, B., Chen, J., and Lu, B. (2005). Genetic diversity and conservation of common wild rice (Oryza rufipogon) in China. Plant Species Biol. 20, 83-92. doi: 10.1111/j.1442-1984.2005.00128.x

Sun, X., Liu, D., Zhang, X., Li, W., Liu, H., Hong, W., et al. (2013). SLAF-seq: an efficient method of large-scale de novo SNP discovery and genotyping using high-throughput sequencing. PLOS ONE 8:e58700. doi: 10.1371/journal.pone.0058700

Thomson, M. J., de Ocampo, M., Egdane, J., Akhlasur Rahman, M., Godwin Sajise, A., Adorada, D. L., et al. (2010). Characterizing the saltol quantitative trait locus for salinity tolerance in rice. Rice 3, 148-160. doi: 10.1007/s12284-0109053-8

Tian, L., Tan, L., Liu, F., Cai, H., and Sun, C. (2011). Identification of quantitative trait loci associated with salt tolerance at seedling stage from Oryza rufipogon. J. Genet. Genomics 38, 593-601. doi: 10.1016/j.jgg.2011. 11.005

Todaka, D., Nakashima, K., Shinozaki, K., and Yamaguchi-Shinozaki, K. (2012). Toward understanding transcriptional regulatory networks in abiotic stress responses and tolerance in rice. Rice 5:6. doi: 10.1186/1939-8433-5-6
Wan, L., Zhang, J., Zhang, H., Zhang, Z., Quan, R., Zhou, S., et al. (2011). Transcriptional activation of OsDERF1 in OsERF3 and OsAP2-39 negatively modulates ethylene synthesis and drought tolerance in rice. PLoS ONE 6:e25216. doi: 10.1371/journal.pone.0025216

Wang, C., Hu, S., Gardner, C., and Lübberstedt, T. (2017). Emerging avenues for utilization of exotic germplasm. Trends Plant Sci. 22, 624-637. doi: 10.1016/j.tplants.2017.04.002

Yang, T., Zhang, S., Hu, Y., Wu, F., Hu, Q., Chen, G., et al. (2014). The role of a potassium transporter OsHAK5 in potassium acquisition and transport from roots to shoots in rice at low potassium supply levels. Plant Physiol. 166, 945-959. doi: 10.1104/pp.114.246520

Yoshida, S., Forno, D., Cock, J., and Gomez, K. (1976). Laboratory Manual for Physiological Studies of Rice, 3rd Edn. Manila: International Rice Research Institute.

Zamir, D. (2001). Improving plant breeding with exotic genetic libraries. Nat. Rev. Genet. 2, 983-989. doi: 10.1038/35103590

Zeng, L., Shannon, M., and Grieve, C. (2002). Evaluation of salt tolerance in rice genotypes by multiple agronomic parameters. Euphytica 127, 235-245. doi: 10.1023/a:1020262932277

Zhang, F., and Xie, J. (2014). "Genes and QTLs resistant to biotic and abiotic stresses from wild rice and their applications in cultivar improvements," in Rice - Germplasm, Genetics and Improvement, eds W. Yan and J. Bao (Rijeka: InTech), Chapter 02. doi: 10.5772/56825

Zhang, L., Li, Z., Quan, R., Li, G., Wang, R., and Huang, R. (2011). An AP2 domaincontaining gene, ESE1, targeted by the ethylene signaling component EIN3 is important for the salt response in Arabidopsis. Plant Physiol. 157, 854-865. doi: $10.1104 /$ pp.111.179028

Zheng, H., Zhao, H., Liu, H., Wang, J., and Zou, D. (2015). QTL analysis of $\mathrm{Na}^{+}$and $\mathrm{K}^{+}$concentrations in shoots and roots under $\mathrm{NaCl}$ stress based on linkage and association analysis in japonica rice. Euphytica 201, 109-121. doi: 10.1007/s10681-014-1192-3

Zhou, Y., Yang, P., Cui, F., Zhang, F., Luo, X., and Xie, J. (2016). Transcriptome analysis of salt stress responsiveness in the seedlings of Dongxiang wild rice (Oryza rufipogon Griff.). PLoS ONE 11:e0146242. doi: 10.1371/journal.pone.0146242

Conflict of Interest Statement: The authors declare that the research was conducted in the absence of any commercial or financial relationships that could be construed as a potential conflict of interest.

Copyright (C) 2018 Quan, Wang, Hui, Bai, Lyu, Zhu, Zhang, Zhang, Li and Huang. This is an open-access article distributed under the terms of the Creative Commons Attribution License (CC BY). The use, distribution or reproduction in other forums is permitted, provided the original author(s) or licensor are credited and that the original publication in this journal is cited, in accordance with accepted academic practice. No use, distribution or reproduction is permitted which does not comply with these terms. 